\title{
Still waiting for a plausible Humean theory of reasons.
}

10 March 2013. To appear in Philosophical Studies. The final publication is available at http://link.springer.com/article/10.1007/s11098-013-0117-7

Abstract: In his important recent book Schroeder proposes a Humean theory of reasons that he calls hypotheticalism. His rigourous account of the weight of reasons is crucial to his theory, both as an element of the theory and constituting his defence to powerful standard objections to Humean theories of reasons. In this paper I examine that rigourous account and show it to face problems of vacuity and consonance. There are technical resources that may be brought to bear on the problem of vacuity but implementation is not simple and philosophical motivation a further difficulty. Even supposing vacuity is fixed, the problems of consonance bring to light a different obstruction lying in Schroeder's path. There is a difference between the general weighing of reasons and the context specificity of the correct placing of weight on them in deliberation and this difference cannot be fixed by the resources in the account. For these reasons we are still waiting for a plausible Humean theory of reasons.

\section{Introduction}

In his important recent book Schroeder proposes a Humean theory of reasons that he calls hypotheticalism:

Reason: For $R$ to be a reason for $X$ to do $\mathrm{A}$ is for there to be some $p$ such that $X$ has a desire whose object is $p$, and the truth of $R$ is part of what explains why $X$ 's doing A promotes p. (Schroeder 2007a:59)

The general argument of the book has a number of prongs:

1. that opponents of Humean theories of reasons burden Humean theories with commitments and doctrines that those theories need not accept, and that hypotheticalism explicitly rejects (chapters 2 and 3 ) ${ }^{1}$

2. that hypotheticalism does not fall prey to the standard objections that are raised against Humean theories (chapters 5, 6 and 10);

3. that hypotheticalism can give an account of normative reasons that fits the constraints and roles widely expected or desired of such an account (chapters 4 and 7);

4. that hypotheticalism has additional powerful explanatory benefits (chapter 9).

If successful, then, hypotheticalism is a strong theory. Part of the theory is the account of the weight of reasons that Schroeder offers in chapter 7. It plays a significant role in each of the above prongs.

First of all, Schroeder needs his account of the weight of reasons in order to avoid what he calls the doctrine of proportionalism. It seems natural for a Humean theory of reasons to account for the weight of reasons by the strength of the correlate desires. This has the consequence that people with strong but foolish desires have strong reasons for foolish ends, conflicting with our intuition that they do not. Schroeder thinks that for this kind of reason proportionalism is 'not even extensionally correct' and that furthermore it offers

\footnotetext{
${ }^{1}$ I'm indicating in brackets where these prongs are particularly prominent but they also feature in other chapters.
} 
the wrong kind of analysis of the weight of reasons because 'the weight of reasons is a normative matter' (Schroeder 2007a:100). Opponents of Humean theories will agree, but demand of Schroeder what he has to put in its place.

Secondly, Schroeder's account of the weight of reasons plays an important role in answering two standard objections raised against Humean theories of reasons. Humean theories are held to be committed both to too many reasons, because people have desires for things they in fact lack reasons for, and to too few reasons, because people do not desire things that they in fact have reasons for. Schroeder's answer to the first is that we do have all these reasons, but the ones appealed to by opponents are ones that, whilst present, have very little weight (and which for reasons of conversational implicature we do not cite, since Grice's informativeness requirement implies mentioning only weighty reasons). Hence

Hypotheticalism....does owe us at least enough of an account of the weight of reasons in order to make good on the crucial claim necessary for my response to the too many reasons objection to work. (Schroeder 2007a:101)

Schroeder argues that the too few reasons objection also depends on proportionalism and accepts that he needs to account for 'the agent-neutral reasons of morality [that] are equally good reasons for everyone'(Schroeder 2007a:122).

Thirdly, the account of the weight of reasons is needed if hypotheticalism is to do more than explain merely the existence of reasons. Schroeder wishes to accept 'reason basicness: what it is to be normative is to be analysed in terms of reasons' (Schroeder 2007a:193) and such analyses require reasons to have weights. For example, the account of what someone ought to do is often given in terms of doing whatever has most reason: the reasons for it outweigh those against. Schroeder also thinks that proportionalism fails here, because it fails to explain

'why it is a truism that if one reason is weightier than another, it is correct to place more weight on it in deliberation.' (Schroeder 2007a:122)

He intends to explain this correct placement of weight in deliberation in terms of a more general principle about what is correct to do and applying it to the placing of weight in deliberation. What is correct to do in general is determined by the most weighty of the right kind of reasons, where the right kinds of reasons are relative to the activity (see Schroeder 2007a:134-5).

Finally, Schroeder sketches a number of explanatory benefits of hypotheticalism that depend on his account of weight. Based on that account he explains: the Aristotelean doctrine that 'When you desire the right things to the right degree your motivation to act will correspond to the weight of your reasons' (Schroeder 2007a:196); that reasons and their weight are metaphysically unmysterious because they are reducible as the theory sets out; that because of this last and because of the account of what it is to act for a reason, we have moral knowledge, we have wide disagreement and the virtuous (those whose desire to the degree corresponding to the weight of reasons) are more reliable in moral knowledge. For these reasons I think it is clear that Schroeder's account of the weight of reasons is a central part of hypotheticalism, so central that hypotheticalism pretty much stands or falls with that account. Unfortunately, I don't think that the account works.

Schroeder disciplines himself to present his account of the weight of reasons with defined principles and a formal model that is intended to satisfy those principles. I shall call this Schroeder's rigourous account of the weight of reasons. To have undertaken this discipline is a virtue since it means the account is definite enough to forestall the endless interpretations and reinterpretations that broader and more loosely expressed accounts 
allow and in which philosophy can easily founder. Nevertheless, there is a difficulty in analysing such rigourous accounts. ${ }^{2}$

A broader thought may lie behind the account, indeed, is often mentioned in the set up, and that thought can usually be given a range of rigourous expositions. Since we must address the specific principles and the specific formal model offered by an author there is always the possibility that faults we find might be avoided by a different formal model and differently defined basic principles. The problem then is how much attention to give to the broader thought and the other possibilities. The broader thought is almost certain to have something going for it since otherwise it would not motivate our interest in the rigourous account. On the other hand, the claim on our attention is based at least in part in not leaving things broad but making it all definite enough to face the risk of refutation.

In Schroeder's case there has been some discussion of some of the broader thoughts, not just on the weights of reasons but from the whole book, in a symposium in Philosophical Studies (Dancy 2012; McPherson 2012; Schroeder 2012a, b; ShaferLandau 2012). That discussion did not engage at any depth with the rigourous account; consequently, however strong the objections raised in that discussion may have been, there are a number of very good reasons why any such objections cannot be allowed simply to impugn his rigourous account. The objections may not actually bear on the account since we do not offer such accounts as mere interpretations of our looser concepts and thoughts but also as better replacements for them (consider for example the relation between the common and the topological concepts of continuity). Rigourous accounts have virtues and powers that require analysis on their own terms and these may outweigh defects in some of the underlying thoughts. On the other hand, a formal model may give unwarranted rhetorical support, the mere appearance of rigour, if left unexamined.

So there are good reasons to examine closely Schroeder's rigourous account. I shall explain the account and show the problems I detect therein. I shall be focusing entirely on the account in chapter 7 of the book because even though there is an earlier paper on the same topic (Schroeder 2007b), the paper is mostly about locating proportionalism as the basis for the too many and too few reasons objections and offers what he explicitly states is 'only the sketch of a theory' (Schroeder 2007b:127) that is spelt 'out more completely in Slaves of the Passions' (Schroeder 2007b:131 fn 20). That examination takes a lot of space and the space to return to the broader thoughts and to other possibilities for their implementation will be limited.

\section{Structure of Schroeder's solution}

Schroeder takes off from his main reason for rejecting proportionalism: that 'the weight of reasons is a normative matter' (Schroeder 2007a:100). Ally this with what he calls reason basicness (see above) and the weight of reasons must itself be explained in terms of reasons. These thoughts are to be captured in some defined principles, Attractive Idea, Ought, Correct and Right Kind of Reasons (see below). Attractive Idea and Correct (when applied to Attractive Idea) together constitute a definition of the weightier-than relation and their explanatory success as outlined in section 7.2 is 'evidence that we are on the right track' (Schroeder 2007a:136). A problem with that definition is that it is circular. The general intention is that the definition of the weightier-than relation in section 7.3 is independent of the principles and so solves the circularity problem, whilst at the same time it is supposed to 'be able to preserve the nice picture set up in section 7.2' (Schroeder 2007a:138).

\footnotetext{
${ }^{2}$ See also Russell 1918:179 ff. for discussion on these points.
} 
The definition itself is recursive. Having mentioned his reservation about recursive analyses being disjunctive Schroeder then explains that he is less worried than he might be just because his definition yields Attractive Idea as 'something interesting and unified that we can say about the weight of reasons' (Schroeder 2007a:139). On the other hand, the defined principles were also introduced as motivations for

the shape of the analysis of the weightier-than relation that I advocate, and ...spell ... out in section 7.3 (Schroeder 2007a:129)

So the dialectic of chapter 7, and in particular that of section 7.2 and its relation to defining the weightier-than relation in section 7.3, is complex. As I understand it, the resultant broad structure of the solution to the weight of reasons has at its base the recursive definition of the weightier-than relation which definition entails $\mathrm{s}^{3}$ Attractive Idea. Attractive Idea, however, is not a mere entailment but it in turn 'explains for us why the disjunctive account provided by Weight Base and Weight Recursion is nevertheless in a certain way unified'(Schroeder 2007a:141, his emphasis). Finally, Attractive Idea explains both Ought and Correct and the circularity is not a problem because the weightier-than relation was defined at the base rather than at this level.

The nature of this kind of account is that the weightier-than relation is primary and a reason having weight is derived from the weightier-than relation. ${ }^{4}$ For a reason to have weight is for it to stand in the weightier-than relation to other reasons. Alternatively, being perhaps the most general definition of what it is for a reason to have weight derivatively from a weightier-than relation, for a reason to have weight is for it to make some difference to the weightier-than relation standing between some other reasons when it is added to them somehow. ${ }^{5}$

\section{Formal framework}

Schroeder notes some constraints. First he points out that the metaphor of weight can't be cashed out literally as quantities: quantities are generally comparable whereas reasons need not be; quantities can always be added whereas some reasons are not independent and so can't be added; ${ }^{6}$ and if taken as quantities adding over generalises because, for example, my pound of flour and your pound of flour can be added to make two pounds but my reason to dance cannot be added to your reason to dance.

So he sets up a formal framework to represent reasons and their weights along the following lines:

Strictly speaking, I will say, it is not reasons that have weights, but only sets of reasons. Not any old set of reasons has a weight, however. Only sets of reasons for the same agent to do the same thing count. And finally, I will define the weight of a set of reasons not by establishing a correspondence

\footnotetext{
${ }^{3}$ Schroeder uses the words 'yields' and 'predicts' and we will discuss this later.

${ }^{4}$ Although Schroeder doesn't state this explicitly he confirms in a communication as a referee of this paper that this is the natural thing to say.

${ }^{5}$ In terms of the formal model we are about to look at we would get this: A reason, $\langle r, x, a>$, has

weight just in case there exist $Q, V \in S^{S}$ such that $<r, x, a>\notin Q \cup V$ and $[(Q>V$ and $(Q \oplus<r, x, a>\ngtr V$ or $Q \ngtr V \oplus<r, x, a>$ or $Q \oplus<r, x, a>\ngtr V \oplus<r, x, a>))$ or $(Q \ngtr V$ and $(Q \oplus<r, x, a>>V$ or $Q>V \oplus<r, x, a>$ or $Q \oplus<r, x, a>>V \oplus<r, x, a>))]$.

${ }^{6}$ e.g. Schroeder accepts that if $R$ is a reason to $\Phi$ then the existential fact of there being that reason to $\Phi$ is itself a reason to $\Phi$ but obviously adding the two together doesn't increase the weight of reasons to $\Phi$.
} 
between sets of reasons and some further thing — amounts of weight — but by defining a partial ordering on sets of reasons: the weightier than ordering (Schroeder 2007a:126-7)

So formally, we can represent reasons by ordered triples $<R, X, A>$, where $R$ is a reason, $X$ is an agent, and $A$ is an action-type. Let us say that $<R, X, A>$ is proper if $R$ is a reason for $X$ to do $A$, and let $S$ be the set of all such proper triples. So $S$ represents the set of all reasons. Now we can define a new set, $S^{S}$, of 'addable' subsets of $S$. The members of $S$ are subsets of $S$ that are invariant with respect to $x$ and $a[\mathrm{sic}]^{7}$. Intuitively, they are sets of reasons that it makes sense to add up. And then we define a relation on $S^{S} \times S^{S}, \succ$, which represents the weightier-than relation. $\succ$ is irreflexive, antisymmetric, and transitive, and holds between two sets when the reasons in the first set add up to more than the reasons in the second set. (Schroeder 2007a:127)

The internal structure of this kind of formal model that we shall need to attend to is not as clear as it might be from the brief specification and is not drawn out by Schroeder elsewhere. I am therefore going to articulate it before we move on, with a small change of notation which for obvious reasons is more perspicuous.

Let $R$ be the set of all reason type things, $X$ the set of persons and $A$ the set of action types, and let $x, r$ and $a$ be any member of those domains respectively. When we need to speak of several possibly distinct members of a domain we will index the variables or use letters nearby in the alphabet. Then

$$
S \text {, Schroeder's set of reasons, }=\{<r, x, a>:<r, x, a>\text { is proper }\}
$$

What Schroeder means by 'addable subset' is not (as it sounds) that addable subsets can in general be added together. Both $\{<r$, Fred, $a>\}$ and $\{\langle r$, Joe, $a>\}$ are in $S$ but he intends to exclude the addition of different people's reasons. Rather, an addable subset is a set of reasons each of which can be added together - and what you get having added together all of the reasons in the set is that very set. So reason addition is really defined by

$$
\begin{aligned}
& <r_{i}, x, a>\oplus<r_{j}, x, a>=\left\{<r_{i}, x, a>,<r_{j}, x, a>\right\} \\
& <r_{i}, x, a>\oplus\left\{<r_{j}, x, a>, \ldots .,<r_{n}, x, a>\right\}=\left\{<r_{i}, x, a>,<r_{j}, x, a>, \ldots .,<r_{n}, x, a>\right\}
\end{aligned}
$$

The item on the RHS is the kind of thing Schroeder is calling a subset of addable reasons but it would have been better if he had called them subsets of added reasons and that is what we shall do.

$$
\text { Let } T_{x, a}=\{<r, x, a>:<r, x, a>\in S\} .
$$

$T_{x, a}$ is the set of all the reasons for $x$ to do $a$. Note that the indexed sets, $\left\{T_{x, a}: x \in X, a \in A\right\}$, partition $\mathrm{S}$. Each of these indexed sets is a maximal set of added reasons since any reasons that are eligible for adding together are members of a single one of these sets and any larger set containing one of these indexed sets will have a member that cannot be added to them. Consequently the power set of $\mathrm{T}_{x, \mathrm{a}}$ gives us the set of all the subsets of added reasons for $x$ to do $a$.

Let $P_{x, a}=$ the power set of $T_{x, a}$.

Then

$$
\text { Let } P_{X}=\cup_{a \in A} P_{x, a}
$$

\footnotetext{
${ }^{7}$ These lower case letters are presumably a typo since Schroeder is using upper case for variables, but it is in line with the notation we'll be using in place of his.
} 


$$
\text { Let } P_{a}=\cup_{\mathrm{x} \in X} P_{x, a}
$$

$P_{X}$ is the set of all the subsets of added reasons for $x . P_{a}$ is the set of all the subsets of added reasons for doing $a . P_{x}$ is usefully remembered as the set of all $x$ 's reasons and all combinations of those reasons and likewise $P_{a}$ is the set of all the reasons to do $a$ and all combinations of those reasons. In a standard abuse of notation, in future appearances of these we will speak of $\left\langle r, x, a>\right.$ as being a member of $P_{x}$ or $P_{a}$ when strictly speaking it is the unit set, $\{<r, x, a>\}$, that is the member.

Finally Schroeder's $S^{S}$ is

$$
S^{S}=\cup_{x \in X, a \in A} P_{X, a}=\cup_{x \in X} P_{X}=\cup_{a \in A} P_{X}
$$

So $S^{S}$ is the set of all the subsets of added reasons. Note that $S^{S} \backslash \varnothing$ is partitioned by each of $\left\{P_{x, a} \mid \varnothing: x \in X, a \in A\right\},\left\{P_{x} \mid \varnothing: x \in X\right\}$ and $\left\{P_{a} \mid \varnothing: a \in A\right\} .{ }^{8}$ We'll call $\left\{P_{x} \mid \varnothing: x \in X\right\}$ the partitioning by persons and $\left\{P_{a} \backslash \varnothing: a \in A\right\}$ the partitioning by acts. The weightier-than relation, $\succ$, is the subset of $S^{S} \times S^{S}$ :

$$
\succ=\left\{<Q, V>: Q, V \in S^{S}+\text { constraint on } Q \text { and } V\right\}
$$

where constraint amounts to the substantive definition of the weightier-than relation, a definition which must result in $>$ satisfying the partial order axioms (and any other formal constraints that we think apply). I put it like this rather than including the partial order axioms in constraint (which is how Schroeder puts it) because being a partial order is a truth about reasons that a formal model must satisfy and including the axioms in constraint misrepresents this fact as something internal to the model or part of the substantive definition.

Strictly speaking, the status of this formal model is for $S$ to be the extension of the reason relation, defined as a relation that holds between reasons, persons and actions and for $>$ to be the extension of the weightier-than relation on reasons. Schroeder doesn't worry about regimenting definitions across relations and their extensions. This results in a certain amount of abuse of notation, perhaps particularly speaking of both $r$ and $\langle r, x, a>$ as reasons. In some places we will need to disambiguate but otherwise we won't worry either.

Clearly this framework can be used to furnish models of many different theories of reasons by their definition of constraint. When the formal model is fully defined to give us a model of Schroeder's account of the weight of reasons, constraint will be his substantive definition of the weightier-than relation, $\succ$, on reasons. So this definition distinguishes his formal model from others that could be formulated in the same formal framework. From here on when speaking of $\succ$ we speak of Schroeder's relation exclusively.

Schroeder's definition must not be inconsistent, neither internally nor with the partial order axioms. It must make it determinate for each $Q, V \in S^{S}$ whether the pair $\langle Q, V\rangle$ is in $\succ$ and it must not through some defect result in the vacuity of $\succ$ (e.g. it is no good if $\succ=\varnothing$ ). Then $\succ$ must be consonant with its roles in the defined principles and $\succ$ in combination with those principles must also be consonant with features appealed to in those principles: with facts about reasons, their weight and the correctness of placing

\footnotetext{
${ }^{8}$ It might be thought that $\varnothing$ shouldn't be a member of $S^{S}$ but Schroeder's definition of $S^{S}$ entails it and his recursive definition of the weightier-than relation requires it to be so. I believe any problems can be technically ironed but as is evident here, it's presence complicates the partitions.
} 
weight on reasons in deliberation. We shall now look in detail at the recursive definition and then I shall show that Schroeder has problems with both vacuity and consonance.

\section{The recursive definition}

Let us now see the problem that the recursive definition is to solve. The Attractive Idea given in section 7.2 is:

Attractive Idea For $R$ to be a weightier set of reasons to do $A$ than $S(R \succ S)$ is for it to be correct to place more weight on $R$ than on $S$ in deliberation about whether to do A. (Schroeder 2007a:130) ${ }^{9}$

Schroeder doesn't say it but we must take $R$ and $S$ to be in $S^{S}$ and for the reasons given in the footnote I will be using ' $D$ ' and ' $E$ ' to refer to such sets of reasons. Then we have

Correct For it to be correct to do $A$ is for it to be the case that $S_{A} \succ S_{\sim A}$.

(Schroeder 2007a:134) ${ }^{10}$

where each of these sets of reasons, $S_{A}$ and $S_{\sim A}$, has been defined as sets of reasons of the right kind, which in this case Schroeder intends to be reasons shared by everyone engaged in deliberation (based on his definition of Right Kind of Reasons, see below).

The circularity of defining the weightier than relation, $\succ$, by Schroeder's principles Attractive Idea + Correct is fairly evident since Attractive Idea defines $\succ$ in terms of correctness of placing weight in deliberation and Correct defines the latter in terms of $\succ$ standing between sets of reasons (of the right kind for placing weight in deliberation). Schroeder offers to reveal the circularity thus:

start by taking the Attractive Idea, and substituting in the analysis provided by Correct, in order to make the circularity explicit:

Better For set of reasons $A$ to be weightier than set of reasons $B$ is for the set of all the (right kind of) reasons to place more weight on $A$ to be weightier than the set of all the (right kind of) reasons to place more weight on B. (Schroeder 2007a:138)

I am now going to extend our formal notation, for reasons that will become evident shortly when we investigate the regress in Schroeder's recursive definition. We will be meeting complexly nested propositions whose expression without these symbols would be immensely cumbersome.

Let $D, E$ be in $S^{S}$. Let $d>e$ be the act of placing more weight on $D$ than on $E .{ }^{11}$ Let $\mathrm{W}_{d>e}$ the set of all reasons of the right kind to do $d>e$. Let $d<e$ be the act of placing more weight on $E$ than on $D$. Let $\mathrm{W}_{d<e}$ be the set of all the reasons of the right kind to do $d<e$. Then Better amounts to

$$
\text { Better For } D>E \text { is for } W_{d>e} \succ W_{d<e}
$$

\footnotetext{
${ }^{9}$ As you can see Schroeder uses ' $R$ ' for a set of reasons when he has already used that to stand for a reason, and uses ' $S$ ' for a set of reasons that is a subset of $S$ as earlier defined. Later he uses ' $A$ ' for a set of reasons when earlier it stood for an action. For perspicuity throughout the discussion I shall generally correct these and other notational flaws, including his use of capital letters as variables, with the terminology already introduced.

${ }^{10} S_{A}$ is a set of reasons for doing act $A$ and $S_{\sim A}$ is a set of reasons for not $\operatorname{doing}$ act $A$.

${ }^{11} \mathrm{I}$ am using ' $d>e$ ' as the name of an act type.
} 
It is evident that this is circular and entails a vicious infinite regress, since $D \succ E$ requires a metaphysically prior $\mathrm{W}_{d>e} \succ \mathrm{W}_{d<e}$ which in turn requires a prior pair, and so on. Schroeder's proposed solution is to

improve on Better by making it recursive:

Weight Base One way for set of reasons $A$ to be weightier than set of reasons $B$ is for set $B$ to be empty, but $A$ non-empty.

Weight Recursion The other way for set of reasons $A$ to be weightier than set of reasons $B$ is for the set of all the (right kind of) reasons to place more weight on $A$ to be weightier than the set of all the (right kind of) reasons to place more weight on $B$.

Weight Recursion is basically just Better. Weight Base gives us an independent way for one set of reasons to count as weightier than another, getting us out of the imminent conceptual and explanatory regress.

(Schroeder 2007a:138)

Formally

For $D \succ E$ is either for

Base axiom for $\succ: D$ to be non-empty and $E$ empty, or for

Recursion axiom for $\succ: W_{d>e} \succ W_{d<e}$.

The idea that Schroeder has about his recursive definition is that it is supposed to allow the regress that results from following Better to ground out at some point with a nonempty set outweighing an empty set.

Weight Base simply tries to characterize what it takes for things to stop, in this way - or rather, for an explanation of the weight of some reason ultimately to get started. Weight Recursion tells us how, once it is started, it continues to proceed. (Schroeder 2007a:138-9)

So in general $D \succ E$ waits on $W_{d>e} \succ W_{d<e}$, which in turn waits on $W\left(W_{d>e}>W_{d<e}\right) \succ W\left(W_{d>e}<W_{d<e}\right),{ }^{12}$ which in its turn waits on $W\left(W\left(W_{d>e}>W_{d<e}\right)>W\left(W_{d>e}<W_{d<e}\right)\right) \succ W\left(W\left(W_{d>e}>W_{d<e}\right)<W\left(W_{d>e}<W_{d<e}\right)\right)$ and so on.

Schroeder's idea is that eventually one of the sets on the RHS will be empty whilst the corresponding one on the LHS will be non-empty. So if, for example, $W\left(W_{d>e}>W_{d<e}\right)$ is non-empty and $W\left(W_{d>e}<W_{d<e}\right)$ is empty then by the base axiom $W\left(W_{d>e}>W_{d<e}\right) \succ$ $W\left(W_{d>e}<W_{d<e}\right)$ hence $W_{d>e}>W_{d<e}$ follows by the recursion axiom, whence $D>E$ follows by the recursion axiom.

\footnotetext{
${ }^{12}$ I have extended our notation in a way that is, I hope, intuitive. $W_{d>e}>W_{d<e}$ is the act of placing more weight on $W_{d>e}$ than on $W_{d<e}$ and $W\left(W_{d>e}>W_{d<e}\right)$ is the set of all the right kind of reasons to do so.
} 


\section{Non-standard recursion}

Note first of all that the axioms alone provide no guarantee that we ever meet empty sets and hence no guarantee that the regress grounds out in the base axiom. Contrast this with the way recursive definitions standardly work. For example, consider the definition of addition on the natural numbers from the successor function, $s$, that maps each number to the next one.

Base axiom: $n+1=s(n)$

Recursion axiom: $n+s(m)=s(n+m)$

Since for all natural numbers, $m, s(m)$ is finitely many steps from 1 , finitely many applications of the recursion axiom ( $m-1$, in fact) will get us to the base axiom. For example

$n+4=s(n+3)=s(s(n+2)=s(s(s(n+1))$ by recursion

and now we can apply the base axiom to get

$=s(s(s(s(n)))$, e.g. if $n=1$ the answer is $s(s(s(s(1)))$ which is 5 , since

5 just is the successor of the successor of the successor of the successor of 1 .

This recursive definition of addition guarantees that the addition of any two numbers has a sum because of the nature of the recursion axiom. The recursion axiom, whilst circular, institutes a single step backwards towards the base axiom when there are only finitely many such steps needed to get there. Then the base axiom defines addition at the base in terms of the successor function, thereby removing the circularity.

So standard recursive definitions have a recursion axiom showing how to work back towards the base axiom. Schroeder's recursion axiom does not do that. Schroeder confirms in communication this difference. He is nevertheless confident that his definition captures 'the most important basic structural fact for any account of the weight of reasons to capture' (Schroeder 2012c), a structural fact that he believes is also manifest in the well known non-monotonic cases from epistemology of defeaters and defeaters of defeaters. So there is an interesting broader thought lying behind the recursive definition, that weight involves regression. That thought may be right but, as already mentioned, a commendable virtue of the book's theory of the weight of reasons is precisely not to have left matters at that level but to commit itself to testable definitions. The problems that I am about to elucidate are problems specific to the recursive definition Schroeder offers. If Schroeder is right in the broad thought then they are also problems that any structurally similar account of weight must avoid.

\section{Vacuity}

So Schroeder's recursion axiom does not implement a step back towards the base axiom. As a result, for all we know from the recursive definition of weight we get nothing more than what is promised by the base axiom:

$$
>=\left\{<Q, \varnothing>: Q \in S^{S} \backslash \varnothing\right\} \text {. }
$$

I take it that if $\succ$ is trivial in this way it would amount to the definition being vacuous. Only on the crucial additional assumption that there is a pair from $S^{S} \backslash \varnothing$ for which following the regress created by the recursion axiom results in eventually bumping into a non-empty/empty pair is $\succ$ non-vacuous.

Schroeder motivates the thought that we get to an empty set by reminding us of Lehrer and Paxson's Tom Grabit case, in which we see Tom running from the library with a book. So we have evidence that he stole it, but then Mrs Grabit, his mother, says Tom was 
away but his twin John was in the library, which is an undercutting defeater of knowing Tom stole, which in turn can be undercut by Mrs Grabit being 'a compulsive and pathological liar' (Lehrer and Paxson 1969:228), and so on. As we run down the sequence of defeaters whether we know or don't know alternates with each one. When we inspect putative sequences of defeaters the nature of each member of the sequence is a readily graspable fact and on that basis it seems evident that they just do run out: it doesn't take long to get to the end of all the relevant facts. So here it is intuitive that the defeaters run out at some point making it determinate whether we know. On some internalist views even if the sequence itself were an infinite regress it would not matter because the finitude of our minds guarantees that we possess only finitely many. The determinacy of whether we know is determined by the finitely many of the sequence of defeaters that we possess. Yet the analogy is not exact enough.

Schroeder could not take the internalist way out of an infinite regress. Whether $D \succ E$ does not depend on how much of the regress above it we know, but on the nature of that regress itself. So it is essential for him that the regress be finite.

As illustrated by the Grabit case, it is plausible that the sequence of defeaters concerning any putative item of knowledge is in fact finite, so suppose we take it that it is this finitude and its effect that is to be carried over by analogy. This now achieves far too much. For if we can be sure of meeting an empty set in the regress, and an empty set suffices for determinacy, this means that for all $D, E$ in $\mathrm{S}^{\mathrm{S}}$ we can be sure that either $D \succ E$ or $E \succ D$, which means that $\succ$ is a total order when it should be only a partial order.

Although in the Grabit case the sequence of defeaters merely coming to an end after finitely many suffices for the determinacy of knowledge, the regress coming to an empty set does not suffice. It has to come to an empty set in the right way, namely we need an empty set and a non-empty set at the same level. So even if the analogy makes it plausible that we'll meet an empty set eventually, it does nothing to make it plausible that we'll meet it in the right way.

At this point it might be thought, well, fine, so when we meet empty sets in the right way we've got weighing and when we don't we haven't so whether $D \succ E$ is determinate on that basis. This, however, is just a restatement of where the recursive definition leaves us. What we needed was to prove rather than merely assume that $>$ is non-vacuous. All the Grabit case achieves by analogy so far is to underline the form of the solution that is needed and to prove too much or too little.

Let us look directly at whether the material Schroeder supplies makes plausible that $\succ$ is non-vacuous. In the Grabit case inspection of the sequence of facts makes it plausible that they run out. Does a similar inspection of the contents of Schroeder's regress make it plausible that it runs out at least sometimes in the right way, thereby making $\succ$ nonvacuous? I believe not. The reason is that the contents of Schroeder's regress are almost incomprehensible almost immediately.

Just try to spell out even only the LHS of the antecedent we get after merely two applications of the recursion axiom, $W\left(W_{d>e}>W_{d<e}\right) \succ W\left(W_{d>e}<W_{d<e}\right)$.

Two Steps Antecedent: $W\left(W_{d>e}>W_{d<e}\right)$ is the set of all the reasons there are of the right kind to place more weight on the set of all the reasons there are of the right kind to place more weight on D than $E$ than on the set of all the reasons there are of the right kind to place more weight on $E$ than D.

The problem here is not only that it is hard to make sense of but that it is this hard to make sense of at only the second step. Schroeder is essentially relying on an intuition motivated 
by the Grabit case that somewhere up there we bump into a non-empty/empty pair. But the disanalogy here is extreme and significant: the members of the Grabit regress are readily graspable facts and these are incomprehensible obscurities. I think it is doubtful we have intuitions about what is going on that we can rely on. Trying to grasp the nature of the reasons in the set of Three Steps Antecedent: $W\left(W\left(W_{d>e}>W_{d<e}\right)>W\left(W_{d>e}<W_{d<e}\right)\right)$, it is quite unclear to me what such things would be even in a thorough going nonnaturalist Platonism for reasons, let alone when they have to be Humean reasons.

We must remember that all these reasons must be explicable in terms of Schroeder's definition of a reason (see again Reason above and on Schroeder 2007a:59). Again, very few steps up the regress and it is hard to see how we have things that can be explained in terms of promoting the object of a desire. They must also be partly explicable in terms of placing weight in deliberation (pace Attractive Idea) but something that cannot be mentally grasped cannot be weighed in deliberation and hence there can't be such reasons making it correct to place weight in deliberation.

Now it might be thought that this last point helps Schroeder, and perhaps it does, since it does at least weigh in on the side of meeting empty sets. The problem as it seems to me, however, is that if it does help him that much it seems likely that the emptiness arises consequent on the level of regress reached, ${ }^{13}$ and that is help of exactly the wrong kind. What we need is a pair of a non-empty and an empty set at the same level but this is promising a pair of empty sets at the same level.

So inspection leads me to think we just don't know what is going on here and without Schroeder making it a great deal clearer what is going on in the regress this is nothing more than the presentation of a purely formal possibility. When we bear in mind the obscurity of the kind of things we are talking about in the regress and bring to mind hypotheticalism's reduction of reasons to explanations by desires, a purely formal possibility isn't enough. It has to be shown that the substance of hypotheticalism's reasons can realize that possibility. Until that is done, the general obscurity leaves it entirely obscure whether this definition will result in $>$ being non-vacuous.

Finally, whilst vacuity is a serious failure, non-vacuity alone achieves little. It does nothing to show that whatever does get into $>$ are the right results for Schroeder, the right results being of two kinds. First, that many or most of the relative weights in the intuitive cases appealed to by opponents of Humean theories of reasons will be explained by this definition. Second, that many or most of the relative weights needed in prongs 2, 3 and 4 of his general argument (see above) are furnished by this definition. Given the obscurity of the contents of the regress it, showing that it gives these right results looks extremely hard.

\section{Fixing vacuity}

The root idea is that Humean theories can get out of the problems of proportionalism if they can explain the weighing of reasons in terms of the correct placement of weight. The incipient circularity is unavoidable if we adhere to reason basicness: that normativity is explained by reasons. So the alternatives are to give up reason basicness or to get a recursive definition to work.

The former requires an additional theory of correctness in weighing. I'm not sure how this could be done in a Humean way but it could certainly be done Platonistically. Reasons could be as Schroeder describes: partial explanations of acts promoting desired

\footnotetext{
${ }^{13}$ likely because the complexity that blocks comprehension rises by level rather than across $\succ$ at a single level.
} 
ends whilst their weights are irreducible matters of objective fact. Reason is the faculty that attends to reasons and their weights. When we are rational we place weight in accordance with the objective weights. This would amount to a Humean non-naturalism, for many an unattractive hybrid since both Humeans and non-naturalists are likely to protest the need to include the other half of the hybrid. Schroeder, in particular, would probably think it amounts to abandoning his Humeanism just where he wants it most.

So that leaves only getting a recursive definition to work. The prospects here are unclear. The natural first move is to look for a way of solving the main disanalogy with standard recursion, to find some way for at least some comparisons to step back towards the base axiom. We might start by removing all the reasons in common between the sets, where reasons in common are triples with the same reason type and act type. We would need to explain why reasons are sufficiently atomistic in effect that this can be done as a weight-order-neutral operation: the arguments for organic unities would all stand against this. Worse, this is not going to get us very far in the general case, and other rules for removing introduce other circularity problems. For example, remove subsets $M$ and $N$ from $D$ and $E$ respectively if $\mathrm{M} \succ \mathrm{N}$.

Another route would be to have a definition by induction on the number of reasons. The simplest example might be if, comparing same sized non-empty proper subsets, those of $D$ weigh more than those of $E$ then $D$ weighs more than $E$. The point here is that we could start at the bottom with each unit set of each and the empty set and then build up somehow. The worry would be that we would get path dependent outcomes. A final route would be a combination of recursion and induction, and I think this is the most likely route to a solution, since one could hope to use each to avoid the problems of the other. For example, suppose $D$ and $E$ are sets whose weight we could not build up to inductively. The circularity of the proposal to removes subsets $M$ and $N$ from $D$ and $E$ respectively if $M \succ N$ could be avoided if we could build up inductively to $M \succ N$ —and perhaps for $D$ to outweigh $E$ we would also have to fulfil special conditions, such as all inductive pairs from $D$ and $E$, when weighable, to weigh in one direction rather than another. So there are technical resources to be explored in aid of a satisfactory definition of the weight of Schroeder's reasons accompanied by significant philosophical challenges in motivating particular uses of those resources.

\section{Consonance}

We now turn to consonance: how well $\succ$ works in the defined principles and how as a whole they fit with features appealed to in those principles, with facts about reasons, their weight and the correctness of placing weight on reasons in deliberation. We will assume throughout that $>$ is not vacuous. I shall start by mentioning a couple of isolated issues before turning to a sequence of problems of consonance at the heart of the account: the claim that Attractive Idea constitutes the unity in the account, whether $\succ$ yields (as Schroeder puts it) Attractive Idea, whether Attractive Idea in turn yields Ought and Correct and whether its appearance of allowing the generality of the weight of reasons succeeds. ${ }^{14}$ This will lead us to a significant obstacle to a central thrust of Schroeder's theory, an obstacle that appears in a variety of ways in the sequence and is perhaps the ground of those problems. The obstacle is a difference between the generality of the

\footnotetext{
${ }^{14}$ By the generality of the weight of reasons I mean that any reasons may be weightier than any others. My reasons may be weightier than yours, my reasons to do one thing may be weightier than my reasons to do another and so on. This appears in Schroeder's theory as allowing that any sets of reasons that are members of $S S$ may be weightier than any other.
} 
weight of reasons and the relevance of context to the correct placing of weight on them in deliberation. As we shall see, Attractive Idea tries to bridge this difference but does so unstably. The instability arises from the dropping of an act variable that occurs when Schroeder moves from Attractive Idea to a principle that Schroeder identifies with Attractive Idea called Still Attractive.

\section{Invariance of the weight of reasons}

An entailment of the recursive definition as given is that a member of $S^{S}$ outweighing another does not depend on context: if $D \succ E$ it is fixed that $D \succ E$. So the account entails the invariance of the weight of reasons, but this invariance is controversial. For example, Dancy holds 'Holism in the theory of reasons: a feature that is a reason in one case may be no reason at all, or an opposite reason, in another' (see Dancy 2004:73). That is to say, what in one context is a reason for something in another may be a reason against it. This would mean the outweighing by this reason of another in the first context might be reversed in the second, contrary to invariance. So if Dancy is right then Schroeder's account fails. There may be a way round this within Schroeder's account if the kind of context that Dancy appeals to in showing the varying pollence of reasons can get packed into Schroeder's sets of reasons. There is some awkwardness in doing this, less so for theoretical reasons than for practical reasons, since context generally doesn't look like reasons. $^{15}$

\section{Bad reasons}

It seems that bad reasons are not all the same, some are worse than others, which is to say that even bad reasons have weight. If that is right then a problem with Weight Base is that it implies that any bad reason weighs more than no reason but that strikes me as the wrong way round.

Bad reasons are, of course, controversial. Perhaps what we call bad reasons are not reasons at all, but are rather things we may mistakenly take for a reason (Kolodny could take this view, see Kolodny 2005). In that case their weight is also a mere appearance and need not be explained at all, or can be explained in counterfactual terms. This possibility is why this problem is less clearly well founded than others I raise.

Other alternatives are that bad reasons are reasons but they are the wrong kinds of reasons or motivating reasons. ${ }^{16}$ In a paper discussing the right kind/wrong kind distinction (Schroeder 2010) deploys Correct to explain the distinction (and also fitting attitudes) where because the right kind are reasons shared relative to an activity the wrong kind are reasons that are not so shared. In Schroeder's response to Dancy's discussion (Dancy 2012; Schroeder 2012b) he agrees that motivating reasons are what is believed rather than the belief and seems thereby to accept Dancy's non-factivity for reasons (Dancy 2000:section 6.3). So Schroeder seems to accept the reality of bad reasons.

This may be fixable if we have a prior definition of good and bad reasons (which Schroeder may be able to construct by distinguishing good and bad explanations in his definition of hypotheticalism, see Reason above and on Schroeder 2007a:59). We would need additional base axioms for the two classes of reasons with Weight Base as it stands for the good reasons and another base axiom reversing the direction of $\succ$ for the bad reasons. There might be some problems for the recursion axiom, since now we have weighting chains both 'above' (for good reasons) and 'below' (for bad reasons) the empty

\footnotetext{
${ }^{15}$ For example, cf. Dancy's distinction between reasons and enabling conditions.

${ }^{16}$ One might also take wrong kinds of reasons or motivating reasons as not reasons at all, as in the previous paragraph.
} 
set, but it looks to me like it would still work as it stands. We would also need some motivated definitions of whether good and bad reasons are addable and this might introduce some additional complexity into the structure of $S$. Whether the upshot of all this would serve Schroeder's purposes would require detailed checking.

\section{The importance of Attractive Idea}

I mentioned earlier that Schroeder points out that his recursive analysis is 'ultimately disjunctive' and that he thinks 'disjunctive accounts are rather problematic', presumably for the familiar reasons that such accounts can be accused of being ad hoc. Schroeder says he is 'less worried about the disjunctive nature of' his account than he might be because

the Weight Base/Weight Recursion account yields the result that there is something interesting and unified that we can say about the weight of reasons. And it is simply that:

Still Attractive Set of reasons $A$ is weightier than set of reasons $B$ just in case it is correct to place more weight on $A$ than on $B$.

And that is just the Attractive Idea with which we began, stated now merely as an extensional thesis rather than an analysis. It therefore not only tells us something interesting about what weighty reasons all have in common, but allows us to preserve the predictions outlined in section 7.2' (Schroeder 2007a:139-40)

So the Attractive Idea is no mere way station on the way to the account. It is an important part of the account in two ways: it is what constitutes the unity in the account despite the disjunctivity of the recursive definition and the account goes via the Attractive Idea in order to 'preserve the predictions' of the principles Ought and Correct.

In outlining the structure of his solution above I took it that what Schroeder is either claiming or needs for this yielding of unity and preservation of prediction is that the recursive definition entails the Attractive Idea which in turn entails Ought and Correct. He might dispute this since he does at least motivate Attractive Idea and the principles of Ought and Correct that it predicts (as he puts it) independently. So he might say he is concerned merely with compatibility. To do so would weaken the support that his recursive definition would get from the truth of the principles and since in speaking of prediction he is using the rhetoric of the hypothetico-deductive method (the theory is supported by the truth of its predictions, which are its entailments given auxiliary hypotheses) it sounds as if he wants that support. So we are going to examine whether this intricate relation of $\succ$, Attractive Idea, Ought and Correct works.

Does Attractive Idea solve the disjunctive problem?

Let us start with the principle he says is yielded by the recursive definition: Still Attractive. It is not clear that Still Attractive is in fact the unified thing to be said about the weight of reasons because it is not clear that Still Attractive is true of members of the base pairs set, $\left\{<Q, \varnothing>: Q \in S^{S} \backslash \varnothing\right\}$, which set we get from Weight Base. The problem here is that Still Attractive requires that for $Q$ to be a weightier set of reasons than $\varnothing$ it requires it to be correct to place more weight on $Q$ than on $\varnothing$. Now suppose $Q$ are reasons to do $a$ and we are deliberating whether to do $b$ which is irrelevant to doing $a$. In this case it is not correct to place more weight on $Q$ than on $\varnothing .{ }^{17}$ So although Still Attractive may apply to

\footnotetext{
${ }^{17}$ There is a bullet that could be bitten here, namely to say that it is correct to place some weight on any reason to do anything when deliberating about any other thing but this is at least strongly counter-intuitive.
} 
sets for which Weight Recursion applies, it doesn't apply to all sets of reasons and so it can't be 'what weighty reasons all have in common'.

In fact, the problem just raised gets worse. For suppose we are deliberating whether to do $a$. Now it is correct to place more weight on $Q$ than on $\varnothing$. Still Attractive gives us a contradiction from this and the previous incorrectness: that $Q$ is weightier than $\varnothing$ and $Q$ is not weightier than $\varnothing$. The root of this problem is that, intuitively, the correctness of placing weight on reasons in deliberation is relative to what one is deliberating about, but this fact (let us call it the Relativity of Placing Weight) is not reflected in Still Attractive.

At this point it may appear I have been uncharitable or inaccurate, since Schroeder has clearly identified Still Attractive with Attractive Idea as essentially the same principle ${ }^{18}$ and Attractive Idea has a variable for an act so it is clearly relativised to an act.

The sets of reasons in Attractive Idea are not just any old sets of reasons in $S^{S}$ but reasons to do $a$ and the correctness of placing more weight on one than the other is correctness when deliberating about whether to do $a$. So in proposing Attractive Idea Schroeder was indeed appealing to and capturing the intuition just mentioned. We must therefore read Still Attractive as elliptical in this respect, and since it is supposed to be yielded by the recursive definition we must read the latter as similarly elliptical.

It is clear that Attractive Idea avoids the contradiction because of the act variable. Even if it is not correct to place more weight on $Q$ than on $\varnothing$ in deliberation about whether to do $b$ Attractive Idea does not combine with that to entail that $Q$ is not weightier than $\varnothing$ because it says nothing about what holds for sets of reasons to do $a$ when deliberating about whether to do $b$.

In fact, however, what we have just is seen is the first emergence of a problem about the upshot of the act variables in Schroeder's principles: whether and how the weightier than relation is relativised to acts and whether and how the principles that Schroeder enunciates as relative to acts can be taken in that way without getting into difficulty. The problem is that there are places where Schroeder needs the relativization and others where he needs its absence (which I think is why the variables are there in Attractive Idea but get dropped by the time we get to the recursive definition and Still Attractive) and he can't have it both ways.

The relativization here can be done in two ways. I think that Schroeder does not in fact want the weightier-than relation itself relativised to acts. If he does then the formal model we have looked at is defectively specified since there would have to be a weightier-than relation for each act. That is to say, it is not that $D$ can be weightier than $E$ but that $D$ is weightier-with-respect-to-doing- $a$ than $E$.

The other way that preserves the single weightier than relation is for the defined principle Attractive Idea to set a restriction on the pairs that stand in the weightier-than relation:

Same Action For $D$ and $E$ in $S^{S}, D \succ E$ only if there is a $P_{a} \in\left\{P_{a}: a \in A\right\}$ such that both $D$ and $E$ are in $P_{a}$.

Under this restriction, Attractive Idea does say something unified about all the pairs in $\succ$ because, since $\varnothing$ is in each $P_{a}$, Attractive Idea applies to the base pairs also. Note that in solving these problems by this restriction we have abandoned the generality of the weight of reasons.

\footnotetext{
${ }^{18}$ That is to say, the difference between them is supposed to be only the difference between 'an extensional thesis rather than an analysis'.
} 
Does the recursive definition entail Attractive Idea?

It is evident that Same Action is not entailed by the recursive definition but is an additional constraint on $>$ that Schroeder did not express explicitly. It is also evident that strict attention to the act variable in Attractive Idea and supposing $\succ$ to satisfy Attractive Idea means that Attractive Idea places Same Action as a constraint on $\succ$. Furthermore, the content of Attractive Idea is at least equal to and may exceed Same Action. Since Attractive Idea contains the content of Same Action, which is not entailed by the recursive definition, the latter cannot entail Attractive Idea. So Schroeder's claim that the recursive definition yields Attractive Idea is under threat and cannot be understood as entailment. Can he preserve compatibility?

\section{Is Attractive Idea consistent with the recursive definition?}

The Relativity of Placing Weight causes problems for compatibility. Let us now suppose the action in the triples of $D$ is $a$ and the action in the triples of $E$ is $b$ where $a \neq b$ and each act is irrelevant to the other. In deliberating whether to $a$ it is correct to place more weight on $D$ than on $E$ but in deliberating whether to $b$ it is correct to place more weight on $E$ than on $D$. So by Attractive Idea $D$ are weightier reasons to do $a$ than $E$ and $E$ are weightier reasons to do $b$ than $D$. If the recursive definition of $\succ$ satisfies Attractive Idea then $D \succ E$ and $E \succ D$ but that contradicts the partial order axioms. So either $\succ$ is not a partial order or it is inconsistent with Attractive Idea.

\section{Does Attractive Idea entail Ought and Correct?}

So we have seen that to get round one problem we had to attend strictly to the act variable in Attractive Idea and we have also see that attending strictly to it produces another problem. We will now see a problem for the entailment of the principles Ought and Correct that originates in the same way. We will also see a general problem for $\succ$ satisfying Correct.

We start with the principle Ought that is to be explained by Attractive Idea.

Let $S_{X, A}$ be the set of all of the reasons for $X$ to do $A$ and $S_{X, \sim A}$ be the set of all of the reasons for $X$ not to do $A$. Then...

Ought For it to be the case that $X$ ought to do $A$ is for it to be the case that $S$ $X, A>S_{X, \sim A}$. (Schroeder 2007a:130)

The problem here is, in essence, that because Attractive Idea implies only sets of reasons for a single action can weigh more than each other, $S_{X, A}$ cannot be weightier than $S_{X, \sim A}$ because $\mathrm{A}$ is not the same action as $\sim \mathrm{A} .{ }^{19}$

More formally, $S_{X, A}$ and $S_{X, \sim A}$ are in $S^{S}$. By Same Action, $S_{X, A} \succ S_{X, \sim A}$ only if there is a $P_{a} \in\left\{P_{a}: a \in A\right\}$ such that both $S_{X, A}$ and $S_{X, \sim A}$ are in $P_{a}$. Because $A$ is not the same action as $\sim A$ there are distinct members of $\left\{P_{a}: a \in A\right\}$ with $S_{X, A}$ in one and $S_{X, \sim A}$ in the other, but since $\left\{P_{a} \backslash \varnothing: a \in A\right\}$ partitions $S^{S} \backslash \varnothing$ the intersection of those distinct members contains only $\varnothing$ so unless one or both are $\varnothing$ there is no member of $\left\{P_{a}: a \in A\right\}$ containing both.

Since in the general case neither $S_{X, A}$ nor $S_{X, \sim A}$ is $\varnothing, S_{X, A} \ngtr S_{X, \sim A}$.

Let us now turn to the principle Correct that is to be explained by Attractive Idea.

\footnotetext{
${ }^{19}$ Schroeder's $S_{X, A}$ is our $T_{X, \mathrm{a}}$ but because the indices reduce the potential for confusion (unlike his earlier ambiguous use of ' $S$ ') I have kept his notation here.
} 
Let $S_{A}$ be the set of all reasons of the right kind to do $A$ and $S_{\sim A}$ be the set of all reasons of the right kind not to do $A$. Then ...

Correct For it to be correct to do $A$ is for it to be the case that $S_{A} \succ S_{\sim A}$.

(Schroeder 2007a:134)

The first problem here is that this principle doesn't work because $S_{A} \succ S_{\sim A}$ is necessarily false under the recursive definition of $\succ$. The problem originates in Schroeder's definition of:

Right Kind of Reasons The right kind of reasons to do $A$ are reasons that are shared by everyone engaged in the activity of doing $A$, such that the fact that they are engaged in doing $A$ is sufficient to explain why these are reasons for them. (Schroeder 2007a:135)

Schroeder takes it that all reasons are what he calls agent relational reasons, reasons for someone (see Schroeder 2007a:16 fn.25), which is why the members of $S$ are triples that include a person. We can understand what Schroeder means by a shared reason by considering his definition of

Agent-Neutral [Reason] For $R$ to be a reason to do $\mathrm{A}$ is for $R$ to be an agent-relational reason for all of us to do A.

As I understand Agent-Neutral, context can determine who counts as one of 'us'(Schroeder 2007a:18)

So what this means is that for $r$ to be an agent neutral reason to do $a$ is just for $\langle r, x, a>$ (an agent relational reason) to be in each person $x$ 's set of reasons to do it:

$r$ is an agent-neutral reason to do $a$ just in case for all $x$ in $X,\{<r, x, a>\} \in P_{x}$

This gives us the set of agent neutral reasons as

$$
A N R=\left\{<r, n, a>:<r, n, a>\in S \text { and } \forall \mathrm{x} \in X<r, x, a>\in P_{x}\right\}
$$

Right Kind of Reasons appears to take the context of being engaged in the activity as determining who counts as one of us. So to get $S_{A}$ we take that subset of $X$, call it $U$, which contains the people engaged in the activity of doing $A$, when

$$
S_{A}=\left\{<r, n, a>:<r, n, a>\in S \text { and } \forall x \in U<r, x, a>\in P_{x}\right\}
$$

Assuming that more than one person is engaged in the activity, what this means is that for distinct persons $y$ and $z$ who are in $U$, if $r$ is a right kind of reason to do $A$, both $<r, y, A>$ and $<r, z, A>$ are in the set of the right kind of reason to do $A, S_{A}$. The same goes for $S_{\sim A}$. That means that neither $S_{A}$ nor $S_{\sim A}$ can be in $S^{S}$ since all members of $S^{S}$ are sets of added reasons and a set of added reasons must have the same person in the second place in each triple. Hence $S_{A} \succ S_{\sim A}$ is necessarily false because the relata of $\succ$ have to be in $S^{S}$. Whence Correct entails that it is impossible for it to be correct to do $A$.

This is potentially a serious problem for Schroeder. Just so far as the considerations by which he motivates Correct and Right Kind of Reasons are strong, just that far do they undermine the success of his definition of the weight of reasons. Schroeder doesn't only defend Correct and Right Kind of Reasons in his book. In a later paper he defends the same two principles (see RKR and Correct, Schroeder 2010:38-39)

There is a way round this, namely to reformulate Correct in terms of the set of all the right kind of reasons for a specific person (i.e. for some $x, S_{A} \cap P_{x}$ ), since if they are shared then the weighing for one person should do as well as for any other. The problem now is whether such a reformulated Correct has the same support of our intuitions. The 
appeal of Correct is precisely in part that it is in terms of the set of all the right kinds of reasons, not just some of them, and although one can see how the reformulation gets out of this difficulty it does look a bit ad hoc, since it is motivated purely by the difficulty the theory has got into rather than any prior appeal to relativising Correct to one particular agent.

Granted this solution to the problem, Correct now falls into the same problem that faced Ought. Once again, because Attractive Idea implies only sets of reasons for a single action can weigh more than each other, $S_{A}$ cannot be weightier than $S_{\sim A}$ because $A$ is not the same action as $\sim A$. The formal proof is the same as for Ought.

So, having seen earlier where Schroeder needs Attractive Idea rather than Still Attractive, here is where Schroeder needs Still Attractive rather than Attractive Idea. Reading Still Attractive strictly rather than as an ellipsis we have something sufficient for explaining Ought and Correct since it speaks of any old sets of reasons in $S^{S}$.

Fred's reasons can be weightier than mine

We now turn to a further problem over whether the theory allows for the generality of the weight of reasons. Fred's reasons to do something can be weightier than mine so this is something the theory shouldn't exclude. It is excluded by an implication of his rejection of weights as quantities.

Though it makes sense to ask how Ronnie's two reasons to go to the party add up, I do not think that it makes sense to ask how Ronnie's reason to go to the party adds up with Susan's reason to go to the lounge. Ronnie's two reasons to go to the party need to be able to add up in order to outweigh his reason to stay away. But it is simply bizarre to think that Ronnie's reason to go to the party and Susan's reason to go to the lounge can add up in some way, for there is nothing that they would need to outweigh....Since I don't think this makes sense, I think it should be a constraint on an adequate account of the weight of reasons that it rule this out. (Schroeder 2007a:126)

What he is saying here is that speaking of adding reasons is really just a way of speaking of what really matters: whether some 'added' reasons outweigh other 'added' reasons or not. Turning to the formal account, it is just a way of speaking of whether sets of added reasons stand in the weightier-than relation. So it is standing in the weightier-than relation that is primary and whether specific reasons can be added is entailed by them belonging to distinct sets of reasons that do stand in that relation. This means that if we are to rule out the reasons of different persons being added up then sets of reasons of different persons cannot stand in the weightier-than relation. But that means that Fred's reasons to do something cannot be weightier than mine nor vice versa.

Now that argument is disputable depending on exactly what is required for the constraint to be captured formally. On this interpretation I have taken the weightier-than relation being primary amounting to it being necessary and sufficient for addition but the argument can be avoided if primality can be captured by necessity alone.

There is, however, another way in which Schroeder's theory may rule out Fred's reasons being weightier than mine. Making $\succ$ depend on correctness in deliberation may rule this out. If I am deliberating about whether to do something I can't make any direct use of the thought that Fred has a heavier reason to do it than I do. For I am deliberating about whether $I$ will do it and his reason to do it as such is not a reason in the way that 
mine is for me. ${ }^{20}$ The way his having a heavier reason can be a reason bearing on me doing it requires not his reason, $r$, but the fact of his reason, $<r$, Fred, $a>$, being a reason for me for some reason or other, such as that I can persuade him or predict his action knowing his reason. So his triple in the set of his reasons is $\langle r$, Fred, $a>$ but that is not the kind of thing that I can weigh, whereas the kind of thing I can weigh is my triple $<<r$, Fred, $a>$, me, $a>$, but that can't be in his set of reasons. Therefore Fred's reason is not something whose weight I weigh against mine in deliberation on whether I do something, but rather, that he has that reason is a reason for me. So I can't weigh Fred's reason as such at all, so a fortiori I can't weigh it more heavily than mine in deliberating what to do. Consequently (and assuming that being correct requires being possible) in Schroeder's theory Fred's reasons cannot weigh more than mine.

It may appear that we can get out of this problem by drawing attention to the apparent agent neutrality of the reasons in Attractive Idea, since then I deliberate not on my reasons but just on reasons to do something, among which might be reasons that I do it and reasons that Fred does it. This would work if Schroeder had agent neutral reasons as a non-Humean might have them. There, all reasons are agent neutral and an agent relational reason for me to do something is just an agent neutral reason that I do it. ${ }^{21}$ But for Schroeder it is exactly the other way round. All reasons are agent relational and speaking of an agent neutral reason to do something is speaking of a complicated fact about a relation among agent relational reasons, for example, that $<r, m e, a>$ is an agent neutral reason I have just in case $<r, m e, a>$ is in $P_{m e}$ and $\forall x \in X<r, x, a>\in P_{x} .{ }^{22}$

So we haven't got much further since for reasons already given Fred's agent neutral reason, $<r$, Fred, $a>$, still cannot figure in my deliberations in the right way for me to place weight on it. It is true that we each have a 'copy' of each others agent neutral reasons so some work can be got done. Unfortunately it still doesn't get us what we were looking for. Without a variable for the agent to relativise the correct placement of weight in Attractive Idea the ordering of my agent neutral reasons and his must be same so we still can't get his reasons being weightier than mine.

At this point, having been reminded of the nature of Schroeder's agent neutral reasons, it is tempting to think that we can at least manage a simulacrum of Fred's reasons being weightier than mine. There is a representation of his agent neutral reasons in my reasons because of the 'copies' of each other's agent neutral reasons that we have and in that way his reasons could get weighed against mine. That is true, so far as it goes, which is not far enough because what makes Fred's reasons weightier than mine need not be agent neutral reasons but reasons he has that I don't.

Finally, if the apparent agent neutrality of Attractive Idea is taken strictly we would have a failing of the theory on exactly one of the motivating issues for Schroeder. It would mean that only agent neutral reasons are defined to have weights.

\footnotetext{
${ }^{20}$ It might be that because $<r$, Fred, a $>$ and $<r$, me, a $>$ are in $\mathrm{S}, r$ is a reason for both of us but that is beside the point, not least because that is not the general case and we are trying to allow for Fred's reasons to be heavier than mine. In Schroeder's theory that requires the set including $<r$, Fred, a $>$ to have more weight placed on it that the set including $<r, \mathrm{me}, \mathrm{a}>$.

${ }^{21} \mathrm{I}$ 'm being a bit short here, but an example might be a general agent neutral reason for parents to look after their children and then because I am the parent of this child there is an agent neutral that I feed him but no agent neutral reasons of this kind that anyone else do.

${ }^{22}$ Alternatively, you might take an agent neutral reason to do $a$ to be the set $\{<r, n, a>:<r, n$, $a>\in S$ and $\left.\forall \mathrm{x} \in X<r, x, a>\in P_{x}\right\}$ but that is not going to help at all since it is not in $S^{S}$ and so can't be a relatum of $\succ$.
} 


\section{Correctness of me or Fred placing weight}

If we suppose, contra the arguments just given, that the account does allow the full generality of the weight of reasons and that I can weigh Fred's reasons against mine in deliberation, then one final problem emerges. We saw a problem for Still Attractive because of its absence of an act variable. Essentially the same problem now arises for Attractive Idea because of its absence of a person variable. Suppose that $D$ and $E$ are sets of reasons to $a$ and that $D$ is in $P_{m e}$ and $E$ is in $P_{F r e d}$ and that neither of our reasons bear on each others doing or not doing $a$. Then, assuming that I can weigh Fred's reasons against mine in deliberation, it is correct for me to place more weight on $D$ than on $E$ so by Attractive Idea $D \succ E$ and it is not correct for Fred to place more weight on $D$ than on $E$ so $D \ngtr E$. Also it is correct for Fred to place more weight on $E$ than on $D$ so $E \succ D$. So we have a contradiction and also inconsistency with the partial order axioms. We can block these if we have a person variable in Attractive Idea, which would amount to placing this constraint on $\succ$ :

Same Person For $D$ and $E$ in $S^{S}, D \succ E$ only if there is a $P_{x} \in\left\{P_{x}: x \in X\right\}$ such that both $D$ and $E$ are in $P_{x}$.

The price of this constraint is to return, once again, to the theory excluding Fred's reasons to do something being weightier than mine and to the theory thereby failing to allow the generality of the weight of reasons.

\section{Fixing consonance}

The dissonances we have seen subsequent to the first two isolated ones are, I think, fairly characterised as dissonances in the heart of the rigourous account. They have their root in what I called the Relativity of Placing Weight. I concede that in developing these dissonances I have made blunt and brute appeals to intuitions about the context relativity of the correctness of placing weight. As always, such appeals to intuition are up for dispute. Nevertheless, I think they have prima facie plausibility, as do Schroeder's principles. What I think the dissonances bring to light, in part because of the definiteness of Schroeder's principles, is some hitherto unclear conflicts between our intuitions about these relativities of correct weighing to acts and persons and intuitions about the generality of the weight of reasons.

Attractive Idea formulates weightier-than and the correctness of placing weight in deliberation relative to an act and is about reasons to do the act. Ought is formulated relative to a person and act and is about all the reasons had by the person for and against that act. Correct is formulated relative to an act and the right kind of reasons for and against the act, reasons shared by everyone engaged in the activity of doing that act. ${ }^{23}$ The recursive definition is given without any such relativities just because where Schroeder wants to end up is with the generality of the weight of reasons.

\footnotetext{
${ }^{23}$ In fact there is a problem with Right Kind of Reasons which manifests in the oddness of saying 'the activity of doing that act', something that gets said because the relativity of Right Kind of Reasons is restricted to a single act variable. It can be shown that this doesn't work but the problem can be fixed by formulating it with both an act and an activity variable: The Right Kind of Reasons to do a certain act are reasons to do it shared by everyone engaged in a certain activity, such that the fact that they are engaged in that certain activity is sufficient to explain why these are reasons for them. Then this gives us Correct Deliberation: the correctness of placing more weight on $D$ than on $E$ is for the reasons to place more weight on $D$ than on $E$ that are shared by everyone deliberating to be heavier than the reasons not to do so that are shared by everyone so engaged, and for the fact they are deliberating to be sufficient to explain why those are reasons for them.
} 
It is no accident that these have been formulated with their various specific relativities. It is in part in virtue of those relativities that they sound right, that they get intuitive support. If instead we start removing or adding in relativities to fix the dissonances we may find our intuitions running the other way.

There is a reason why Schroeder started with Attractive Idea. With the act variable in place and read as elliptical with respect to the who of the reasons being kept fixed (and setting aside the circularity worries over what correct deliberation is), it is exactly where it is intuitively plausible that he can start. Recall his reduction of reasons to being parts of explanations of why a person in doing something is promoting something they desire. Given this reduction it is initially comprehensible that for a specific person in deliberating on whether to do something it would be correct for him to place more weight on some reasons than on others to do it. It may bear further explanation, but possibilities for such explanation seem near to hand: for example, one reason shows the promotion to be more likely or more direct. So even though Schroeder's official story via the correctness of placing weight has an incipient circularity the possibility of these kinds of explanations show us the kind of thing that might ground it (thereby thinking that something else is to hand to replace the obscurities of the regress in the recursive definition). On the one hand they give plausibility to weighing for Schroeder's reasons without having to appeal to the strength of desires. On the other hand they show what kind of thing the right kind of reasons for placing more weight on one set than another might be. So we have something with which to mollify the circularity worry.

If, by contrast, he started with something in terms of it being correct for that specific person to place more weight on some reasons to do it than some other reasons not to do it, in the general case we are comparing explanations of why doing something is promoting something they desire with explanations of why not doing it is promoting some other thing they desire. Given Schroeder's rejection of proportionalism (so we can't explain correctness of placing more weight on one that the other by strength of desire) it is difficult to see a basis for the comparison just because the underlying desires that explain the reasons are distinct. Now all we have to look to is Correct and we have nothing to set against its incipient circularity and the obscurity we have seen in the recursive definition.

We might instead appeal to the brute plausibility (in a specific case) of being heavier making it being correct to place more weight on some reasons to do it than some other reasons not to do it. My worry then would be either that the explanation is going the wrong way or that behind it is the wrong intuition for Schroeder's purposes. Going the wrong way, in that the correctness of placing weight is being explained by an intuition about the reasons for doing it being heavier than the reasons not to do it. The wrong intuition, in that it is if you ought to do it then it is correct to place more weight on the reasons for it than against it. And this is no good for Schroeder because he wants to analyse 'ought' in terms of the weight of reasons, not the weight of reasons in terms of 'ought'. So that is why he didn't start here.

The nexus is the contrast between Attractive Idea, with relativities in place, and Still Attractive, without them, as what is yielded by the recursive definition. The relativity of correctly placing weight in deliberation sounds right in Attractive Idea but that blocks explanation of Ought and Correct. Omitting the relativity of correctly placing weight, as in Still Attractive, gets us the explanation of Ought and Correct but fails to solve the disjunctive problem and falls into contradiction.

What is going on here is that Attractive Idea and Still Attractive are trying to face both ways, to meet incompatible demands: on the reasons side to get the general weighing of sets of reasons, on the correct placing of weight side to get the relativity to act and person. 
Attractive Idea sounds right when we focus on the correctness of placing weight, but when we turn our attention to weighing reasons in general the restriction to reasons for a specific act looks dispensable. Hence we accept Still Attractive on the intuition that supported Attractive Idea. But then if we examine Still Attractive while focusing again on the correctness of placing weight, even if sets of reasons can generally outweigh one another, it is not clear that that alone makes it correct to place more weight on one set of reasons rather than another without reference to the context of deliberation, what act is being deliberated on and who is doing the deliberation. The truism to which Schroeder appealed earlier, that 'if one reason is weightier than another, it is correct to place more weight on it in deliberation.' (Schroeder 2007a:122), has some implicit contextual restriction on the domain of reasons that get into the antecedent.

So in the Humean framework that Schroeder is promoting, there is a gap between where Schroeder starts, sets of agent relational reasons for a specific person to do a specific thing, ${ }^{24}$ and where he wants to get to, weighing sets of reasons in general. ${ }^{25}$ What I think the dissonances have brought to light is that there is an interesting difficulty lying in the way that Schroeder has taken to get across the gap, a difficulty located not so much in the regress but in the relation between reasons outweighing one another and the correctness of placing weight in deliberation. The former is intuitively general whereas the latter is intuitively relative to acts and persons. The former is where he needs to get to, the latter, with their relativities matching his agent relational reasons, is where it seems unproblematic for him to start. In moving from Attractive Idea to Still Attractive whilst claiming identity (modulo the difference between 'an extensional thesis rather than an analysis') Schroeder hasn't overcome the difficulty but sidestepped it. Fixing it would require a deeper understanding of this relation and additional defined principles. In particular it would require a more complex account of the right kind of reasons for deliberation. Schroeder has two papers on the right kind of reasons (Schroeder 2010, 2012d) but I do not see in them material that bears here.

\section{Conclusion}

Someone sympathetic to Humean theories of reasons, such as myself, might wonder whether the kind of approach Schroeder is taking can get round the problems we have seen. The suggestion that weighing is regressive, based in the analogy with defeaters, is interesting. We have seen that Schroeder's implementation of this idea by his recursive definition contains significant obscurities that undermine the support given by the analogy leaving it unclear whether it achieves what it sets out to achieve. Whilst Schroeder's implementation may yet be fixed, it would be at the cost of significant technical complication. Complication alone need not be a heavy fault since I see no reason to expect a neat theory: in general morality is messy and depends intricately on a lot of the particular detail of cases. We should therefore expect the true theory of reasons to be messy in some respects. Unfortunately, technical complication perhaps entails a heavy theoretical burden, since each technical complication requires its own substantive philosophical motivation in Humean terms, or at least, motivation in terms consistent with Humeanism. ${ }^{26}$ Providing such motivations is a significant philosophical challenge in its

\footnotetext{
${ }^{24}$ i.e. sets in $P_{x, a}$ for specific $x$ and $a$.

${ }^{25}$ i.e. sets in $S^{S} \backslash \varnothing$, which is partitioned by each of $\left\{P_{x, a} \backslash \varnothing: x \in X, a \in A\right\},\left\{P_{x} \backslash \varnothing: x \in X\right\}$ and $\left\{P_{a} \backslash \varnothing\right.$ : $a \in A\}$ and which partitionings are the formal ground of the problems.

${ }^{26}$ Schroeder questions whether 'every adjustment would need to be motivated on Humean terms' although agrees that one might be motivated to think this 'by a reading of the somewhat aggressive dialectical posture of the book' Schroeder 2012c.
} 
own right. Against this we might put my earlier remarks about the virtues of rigourous accounts and the support got for such an account by its getting things right despite internal technical complexity. Given the naturalism that Schroeder is aiming at he might be very happy at taking the move often taken by scientists: of recommending replacing our loose common concepts with those of a rigourous theory that is getting a lot right.

Nevertheless, at the moment the account itself is obscure, the nature of the technical fixes that might be needed is unknown and the philosophical motivation of any such fixes is a further and perhaps significant difficulty. The central dissonances discussed are at the heart of the account and appear to be grounded in the relativity of the correct placement of weight, which relativity obstructs the path from the recursive definition to the broader explanatory objectives of the account. I therefore think we are still waiting for a plausible Humean theory of reasons.

\section{References}

Dancy, J. 2000. Practical Reality. Oxford: Oxford University Press.

Dancy, J. 2004. Ethics without Principles. Oxford: Clarendon Press.

Dancy, J. 2012. Response to Mark Schroeder's Slaves of the Passions. Philosophical Studies, 157 (3), pp. 455-62.

Kolodny, N. 2005. Why Be Rational? Mind, 114 (455), pp. 509-63. July 1, 2005. Online at http://mind.oxfordjournals.org/cgi/content/abstract/114/455/509

Lehrer, K. \& Paxson, T., Jr. 1969. Knowledge: Undefeated Justified True Belief. The Journal of Philosophy, 66 (8), pp. 225-37. Online at http://www.jstor.org/stable/2024435

McPherson, T. 2012. Mark Schroeder's Hypotheticalism : Agent-Neutrality, Moral Epistemology, and Methodology. Philosophical Studies, 157 (3), pp. 445-53.

Russell, B. 1918. The Philosophy of Logical Atomism. In Logic and Knowledge: Essays 1901-1950. Ed. Marsh, R. C. London: Unwin Hyman.

Schroeder, M. A. 2007a. Slaves of the Passions. Oxford: Oxford University Press.

Schroeder, M. A. 2007b. Weighting for a Plausible Humean Theory of Reasons. Nous, 41 (1), pp. 138-60.

Schroeder, M. A. 2010. Value and the Right Kind of Reasons. In Oxford Studies in Metaethics. Vol. 5. Ed. Shafer-Landau, R. Oxford: Oxford University Press.

Schroeder, M. A. 2012a. Precis of Slaves of the Passions. Philosophical Studies, 157 (3), pp. 431-4.

Schroeder, M. A. 2012b. Reply to Shafer-Landau, Mcpherson, and Dancy. Philosophical Studies, 157 (3), pp. 463-74.

Schroeder, M. A. 2012c. Reviewer \# 1 Comments.:

Schroeder, M. A. 2012d. The Ubiquity of State-Given Reasons. Ethics, 122 (3), pp. 45788. Online at http://www.jstor.org/stable/10.1086/664753

Shafer-Landau, R. 2012. Three Problems for Schroeder's Hypotheticalism. Philosophical Studies, 157 (3), pp. 435-43. 